\title{
Analysis of the fractional diffusion equations with fractional derivative of non-singular kernel
}

\author{
Mohammed Al-Refai ${ }^{*}$ and Thabet Abdeljawad ${ }^{2}$
}

"Correspondence:
m_alrefai@uaeu.ac.ae
'Department of Mathematical
Sciences, UAE University, P.O. Box
15551, AI Ain, UAE
Full list of author information is
available at the end of the article

\begin{abstract}
In this paper we study linear and nonlinear fractional diffusion equations with the Caputo fractional derivative of non-singular kernel that has been launched recently (Caputo and Fabrizio in Prog. Fract. Differ. Appl. 1(2):73-85, 2015). We first derive simple and strong maximum principles for the linear fractional equation. We then implement these principles to establish uniqueness and stability results for the linear and nonlinear fractional diffusion problems and to obtain a norm estimate of the solution. In contrast with the previous results of the fractional diffusion equations, the obtained maximum principles are analogous to the ones with the Caputo fractional derivative; however, extra necessary conditions for the existence of a solution of the linear and nonlinear fractional diffusion models are imposed. These conditions affect the norm estimate of the solution as well.
\end{abstract}

Keywords: fractional diffusion equations; maximum principle; fractional derivatives

\section{烈 Springer}

\section{Introduction}

Fractional diffusion models (FDM) are generalization to the diffusion models with integer derivatives. In recent years there has been great interest in the study of FDM because of their appearance in modeling various applications in the physical sciences, medicine and biology; see, for instance, [2-10]. Therefore, analytical and numerical techniques have been implemented to study these models. The maximum principle is one of the common tools to study partial differential equations analytically, see [11, 12] for intensive survey and results. In recent years, maximum principles have been developed to study various types of fractional diffusion systems (see [13-22]), and we refer the reader to [23] for the recent development on the theory of fractional differential equations. In [13] and [14], two classes of eigenvalue problems of Caputo fractional order $\alpha, 1<\alpha<2$, were considered. Maximum principles and the method of lower and upper solutions have been developed and used to establish certain existence and uniqueness results of the problems. In [19] and [21] Luchko has developed and implemented maximum principles to study the generalized fractional diffusion equation of Caputo fractional derivative. Existence and uniqueness results were established by the new maximum principles obtained by estimating the fractional derivative of a function at its extreme points. Analogous results were obtained in $[20,22]$ for the fractional diffusion systems of multi-term and distributed order frac-

(c) The Author(s) 2017. This article is distributed under the terms of the Creative Commons Attribution 4.0 International License (http://creativecommons.org/licenses/by/4.0/), which permits unrestricted use, distribution, and reproduction in any medium, provided you give appropriate credit to the original author(s) and the source, provide a link to the Creative Commons license, and indicate if changes were made. 
tional derivatives of Caputo type. Another maximum principle for the linear multi-term fractional differential equations with the modified Riesz fractional derivative of Caputo type was introduced and employed in [24]. The applicability of maximum principles for the linear and nonlinear fractional diffusion systems with the Riemann-Liouville fractional derivative was discussed and proved for the first time by Al-Refai and Luchko in [15], where existence, uniqueness and stability results were established. Analogous results for the fractional diffusion systems with the multi-term and distributed order fractional derivatives of Riemann-Liouville type were obtained in [16] and [18]. In [25] and [26], a maximum principle was used to analyze a type of fractional diffusion equation without an explicit formulation of this principle.

Recently [1] Caputo et al. have introduced a new type of fractional derivative with nonsingular kernel. After then, the authors in [27-29] studied their discrete versions and analyzed the monotonicity properties for the fractional difference operator. Insisting on the importance of having fractional operators with non-singular kernels, later in [30-32] the authors introduced, explored and studied fractional operators of Mittag-Leffler kernels together with their discrete versions.

In this paper, we extend the results presented in [15] for the fractional diffusion equations with the Caputo fractional derivative of non-singular kernel. The rest of this paper is organized as follows. First, we give the basic definitions and results about fractional derivatives with exponential kernels. In Section 2, an estimate of the Caputo fractional derivative of non-singular kernel of a function at its extreme points is deduced in a form of certain inequality. This inequality is then employed to derive a weak and a strong maximum principles for the time-fractional diffusion equation with the Caputo fractional derivative of non-singular kernel. We apply the obtained maximum principles to analyze the solutions of linear and nonlinear time-fractional diffusion models in Sections 3 and 4, respectively. Uniqueness and stability results as well as norm estimates of solutions are obtained. Some illustrative examples are presented in Section 5. Finally, we close up with some concluding remarks in Section 6.

Definition 1.1 ([1,27]) Let $f \in H^{1}(a, b), a<b, \alpha \in[0,1]$, the (left Caputo) fractional derivative in the sense of Caputo and Fabrizio is defined by

$$
\left({ }^{\mathrm{CFC}} D_{a}^{\alpha} f\right)(t)=\frac{B(\alpha)}{1-\alpha} \int_{a}^{t} f^{\prime}(x) e^{\left[-\alpha \frac{(t-x) \alpha^{\alpha}}{1-\alpha}\right]} d x
$$

The associated fractional integral is defined by

$$
\left({ }^{\mathrm{CF}} I_{a}^{\alpha} f\right)(t)=\frac{1-\alpha}{B(\alpha)} f(t)+\frac{\alpha}{B(\alpha)} \int_{a}^{t} f(s) d s
$$

where $B(\alpha)>0$ is a normalization function satisfying $B(0)=B(1)=1$.

Lemma 1.1 ([27]) For $0<\alpha<1$, we have

$$
\left({ }^{\mathrm{CF}} I_{a}^{\alpha \mathrm{CFC}} D_{a}^{\alpha} f\right)(x)=f(x)-f(a)
$$




\section{Maximum principles}

We start with estimating the fractional derivative of a function at its extreme points. These results are analogous to the ones obtained in [33] for the Caputo fractional derivative. We then use these results to establish new maximum principles for linear fractional equations with Caputo fractional derivative of non-singular kernel.

Lemma 2.1 Let a function $f \in H^{1}(0, T)$ attain its maximum at a point $t_{0} \in[0, T]$ and $0<\alpha<1$. Then the inequality

$$
\left({ }^{\mathrm{CFC}} D_{0}^{\alpha} f\right)\left(t_{0}\right) \geq \frac{B(\alpha)}{1-\alpha} e^{-\frac{\alpha}{1-\alpha} t_{0}}\left(f\left(t_{0}\right)-f(0)\right) \geq 0
$$

holds true.

Proof We define the auxiliary function $g(t)=f\left(t_{0}\right)-f(t), t \in[0, T]$. Then it follows that $g(t) \geq 0$, on $[0, T], g\left(t_{0}\right)=g^{\prime}\left(t_{0}\right)=0$ and $\left({ }^{\mathrm{CFC}} D_{0}^{\alpha} g\right)(t)=-\left({ }^{\mathrm{CFC}} D_{0}^{\alpha} f\right)(t)$. Since $g \in H^{1}(0, T)$, then $g^{\prime}$ is integrable, and integrating by parts with

$$
u=e^{-\frac{\alpha}{1-\alpha}\left(t_{0}-\tau\right)}, \quad \text { and } \quad d v=g^{\prime}(\tau)
$$

yields

$$
\begin{aligned}
\left({ }^{\mathrm{CFC}} D_{0}^{\alpha} g\right)\left(t_{0}\right) & =\frac{B(\alpha)}{1-\alpha} \int_{0}^{t_{0}} g^{\prime}(\tau) e^{-\frac{\alpha}{1-\alpha}\left(t_{0}-\tau\right)} d \tau \\
& =\frac{B(\alpha)}{1-\alpha}\left(\left.e^{-\frac{\alpha}{1-\alpha}\left(t_{0}-\tau\right)} g(\tau)\right|_{0} ^{t_{0}}-\frac{\alpha}{1-\alpha} \int_{0}^{t_{0}} g(\tau) e^{-\frac{\alpha}{1-\alpha}\left(t_{0}-\tau\right)} d \tau\right) \\
& =\frac{B(\alpha)}{1-\alpha}\left(g\left(t_{0}\right)-e^{-\frac{\alpha}{1-\alpha} t_{0}} g(0)\right)-\frac{\alpha B(\alpha)}{(1-\alpha)^{2}} \int_{0}^{t_{0}} g(\tau) e^{-\frac{\alpha}{1-\alpha}\left(t_{0}-\tau\right)} d \tau .
\end{aligned}
$$

Since $g(t) \geq 0$ on $[0, T]$, the integral in the last equation is nonnegative, and thus

$$
\begin{aligned}
\left({ }^{\mathrm{CFC}} D_{0}^{\alpha} g\right)\left(t_{0}\right) & \leq \frac{B(\alpha)}{1-\alpha}\left(g\left(t_{0}\right)-e^{-\frac{\alpha}{1-\alpha} t_{0}} g(0)\right)=-\frac{B(\alpha)}{1-\alpha} e^{-\frac{\alpha}{1-\alpha} t_{0}} g(0) \\
& =-\frac{B(\alpha)}{1-\alpha} e^{-\frac{\alpha}{1-\alpha} t_{0}}\left(f\left(t_{0}\right)-f(0)\right) .
\end{aligned}
$$

The last inequality yields

$$
-\left({ }^{\mathrm{CFC}} D_{0}^{\alpha} f\right)\left(t_{0}\right) \leq-\frac{B(\alpha)}{1-\alpha} e^{-\frac{\alpha}{1-\alpha} t_{0}}\left(f\left(t_{0}\right)-f(0)\right),
$$

which proves the result.

Lemma 2.2 Let a function $f \in H^{1}(0, T)$ attain its maximum at a point $t_{0} \in(0, T]$ and $0<\alpha<1$. If $f(t)$ is not the constant function on $\left[0, t_{0}\right]$, then the inequality

$$
\left({ }^{\mathrm{CFC}} D_{0}^{\alpha} f\right)\left(t_{0}\right)>\frac{B(\alpha)}{1-\alpha} e^{-\frac{\alpha}{1-\alpha} t_{0}}\left(f\left(t_{0}\right)-f(0)\right) \geq 0
$$

holds true. 
Proof Since $f(t)$ is not constant, then $g(t)=f\left(t_{0}\right)-f(t) \geq 0$ and not identically zero on $\left[0, t_{0}\right]$. Thus

$$
\int_{0}^{t_{0}} g(\tau) e^{-\frac{\alpha}{1-\alpha}\left(t_{0}-\tau\right)} d \tau>0
$$

Then Eq. (2.3) will lead to

$$
\left({ }^{\mathrm{CFC}} D_{0}^{\alpha} g\right)\left(t_{0}\right)<-\frac{B(\alpha)}{1-\alpha} e^{-\frac{\alpha}{1-\alpha} t_{0}}\left(f\left(t_{0}\right)-f(0)\right),
$$

which proves inequality (2.4).

We now employ the previous results to derive weak and strong maximum principles for the time-fractional differential operator $P_{\alpha}$ of parabolic type defined by

$$
P_{\alpha}(u(x, t))=L(u(x, t))-\left({ }^{\mathrm{CFC}} D_{0}^{\alpha} u(x, t)\right)(t), \quad 0<\alpha<1, t \in(0, T], x \in \Omega,
$$

where $\Omega$ is the rectangular region $\Omega:\{0<x<\ell, 0<t<T\}, L(u)=a(x, t) u_{x x}+b(x, t) u_{x}$, and $a(x, t)>0$. The sides of $\Omega$ are

$$
\begin{aligned}
& S_{1}:\{x=0,0 \leq t \leq T\}, \\
& S_{2}:\{0 \leq x \leq \ell, t=0\}, \\
& S_{3}:\{x=\ell, 0 \leq t \leq T\}, \\
& \text { and } S_{4}:\{0 \leq x \leq \ell, t=T\} .
\end{aligned}
$$

We have the following weak maximum principle.

Theorem 2.1 (Weak maximum principle) Suppose that $u(x, t) \in C^{2}[0, \ell] \cap H^{1}(0, T]$ satisfies the inequality $P_{\alpha}(u) \geq 0$ in the rectangular region $\Omega_{T}=(0, \ell) \times(0, T]$, then

$$
\max _{\bar{\Omega}} u=\max _{S_{1} \cup S_{2} \cup S_{3}} u .
$$

Proof Assume by contradiction that the result is not true, then $u$ attains a maximum at $\left(x_{0}, t_{0}\right) \in \Omega_{T}$ with $u\left(x_{0}, t_{0}\right)=M_{1}$, and $M_{1}>\max _{s_{1} \cup S_{2} \cup S_{3}} u$. Because $u_{x}\left(x_{0}, t_{0}\right)=0$, $u_{x x}\left(x_{0}, t_{0}\right) \leq 0$ and $a\left(x_{0}, t_{0}\right)>0$, we have

$$
\left.L(u(x, t))\right|_{(x, t)=\left(x_{0}, t_{0}\right)}=a\left(x_{0}, t_{0}\right) u_{x x}\left(x_{0}, t_{0}\right)+b\left(x_{0}, t_{0}\right) u_{x}\left(x_{0}, t_{0}\right) \leq 0,
$$

and

$$
\begin{aligned}
\left({ }^{\mathrm{CFC}} D_{0}^{\alpha} u\left(x_{0}, t\right)\right)\left(t_{0}\right) & \geq \frac{B(\alpha)}{1-\alpha} e^{-\frac{\alpha}{1-\alpha} t_{0}}\left(u\left(x_{0}, t_{0}\right)-u\left(x_{0}, 0\right)\right) \\
& =\frac{B(\alpha)}{1-\alpha} e^{-\frac{\alpha}{1-\alpha} t_{0}}\left(M_{1}-u\left(x_{0}, 0\right)\right)>0 .
\end{aligned}
$$

Thus,

$$
P_{\alpha}\left(u\left(x_{0}, t_{0}\right)\right)=L\left(u\left(x_{0}, t_{0}\right)\right)-\left({ }^{\mathrm{CFC}} D_{0}^{\alpha} u\left(x_{0}, t\right)\right)\left(t_{0}\right)<0,
$$

and a contradiction is reached. 
Theorem 2.2 (Strong maximum principle) Suppose that $u(x, t) \in C^{2}[0, \ell] \cap H^{1}(0, T]$ satisfies the inequality $P_{\alpha}(u) \geq 0$ in the rectangular region $\Omega_{T}=(0, \ell) \times(0, T]$ and that $u$ attains a maximum at $\left(x_{0}, t_{0}\right) \in \Omega_{T}$. Then $u\left(x_{0}, t\right)=u\left(x_{0}, t_{0}\right)$ for all $0 \leq t \leq t_{0}$.

Proof For $0<t \leq t_{0}$, assume by contradiction that the result is not correct. That is, $u\left(x_{0}, t\right)$ is not constant on $\left(0, t_{0}\right]$. By inequality $(2.4)$ we have

$$
\left({ }^{\mathrm{CFC}} D_{0}^{\alpha} u\left(x_{0}, t\right)\right)\left(t_{0}\right)>0,
$$

and thus

$$
P_{\alpha}\left(u\left(x_{0}, t_{0}\right)\right)=L\left(u\left(x_{0}, t_{0}\right)\right)-\left({ }^{\mathrm{CFC}} D_{0}^{\alpha} u\left(x_{0}, t\right)\right)\left(t_{0}\right)<0,
$$

which contradicts the assumption of the theorem. Since $u\left(x_{0}, t\right)=u\left(x_{0}, t_{0}\right)$, for all $0<t \leq$ $t_{0}$, and by the continuity of the solution $u(x, t)$, we have $u\left(x_{0}, t\right)=u\left(x_{0}, t_{0}\right)$ for all $0 \leq t \leq t_{0}$, which completes the proof.

\section{Linear fractional diffusion problems}

In this section, we consider the time-fractional initial-boundary value problem

$$
\begin{aligned}
& Q_{\alpha}(u)=L(u)-\left({ }^{C F C} D_{0}^{\alpha} u\right)(t)-f(x, t)=0, \quad 0<\alpha<1,(x, t) \in \Omega, \\
& u(x, 0)=g(x), \quad 0 \leq x \leq \ell, \\
& u(0, t)=h_{1}(t), \quad u(\ell, t)=h_{2}(t), \quad t \in(0, T],
\end{aligned}
$$

where $f(x, t)$ is continuous on $\bar{\Omega}=[0, \ell] \times[0, T]$. We start with the following uniqueness result.

Theorem 3.1 The time-fractional initial-boundary value problem (3.1-3.3) has at most one solution $u \in C^{2}[0, \ell] \cap H^{1}(0, T]$.

Proof Assume that $u_{1}(x, t)$ and $u_{2}(x, t)$ are two solutions of (3.1-3.3), and let $v(x, t)=u_{1}-u_{2}$. Then $v(x, t)$ satisfies

$$
\begin{aligned}
& P_{\alpha}(v)=0, \quad 0<\alpha<1, \quad(x, t) \in \Omega, \\
& v(x, 0)=0, \quad 0 \leq x \leq \ell, \\
& v(0, t)=v(\ell, t)=0, t \in(0, T] .
\end{aligned}
$$

Applying the simple maximum principle will lead to $v(x, t) \leq 0,(x, t) \in \bar{\Omega}$. The above statements hold true for $-v(x, t)$, and thus $-v(x, t) \leq 0$. Hence $v(x, t)=0$ and the result is obtained.

In the following we present essential results to guarantee the existence of a solution to the time-initial-boundary value problem (3.1)-(3.3) and to obtain analytical bound of the solution. 
Lemma 3.1 Consider the fractional initial value problem

$$
\left({ }^{\mathrm{CFC}} D_{0}^{\alpha} k\right)(t)=f(t), \quad k(0)=c, t>0,
$$

where $f(t) \in C[0, T]$. The initial value problem has the unique solution

$$
k(t)=c+\frac{1-\alpha}{B(\alpha)} f(t)+\frac{\alpha}{B(\alpha)} \int_{0}^{t} f(\tau) d \tau
$$

if and only if $f(0)=0$. Moreover, it holds that

$$
\|k(t)\|_{[0, T]} \leq|k(0)|+\frac{1-\alpha}{B(\alpha)}\left(1+\frac{\alpha}{1-\alpha} T\right)\|f(t)\|_{[0, T]} .
$$

Proof By the help of Lemma 1.1, we have

$$
k(t)-k(0)=\left({ }^{\mathrm{CF}} I_{0}^{\alpha}{ }^{\mathrm{CFC}} D_{0}^{\alpha}\right) k(t)=\left({ }^{\mathrm{CF}} I_{0}^{\alpha}\right) f(t)=\frac{1-\alpha}{B(\alpha)} f(t)+\frac{\alpha}{B(\alpha)} \int_{0}^{t} f(\tau) d \tau,
$$

which proves the result.

The result in Eq. (3.5) follows since

$$
\left\|\int_{0}^{t} f(\tau) d \tau\right\|_{[0, T]} \leq\|f(t)\|_{[0, T]} \int_{0}^{t} d \tau=\|f(t)\|_{[0, T]} t \leq\|f(t)\|_{[0, T]} T .
$$

We have the following necessary condition for the existence of solution to Eq. (3.1).

Lemma 3.2 The time-fractional equation (3.1) has a solution only if

$$
(L(u))(x, 0)=f(x, 0),
$$

where $L(u)=a(x, t) u_{x x}+b(x, t) u_{x}$.

Proof We have $\left({ }^{\mathrm{CFC}} D_{0}^{\alpha} u\right)(t)=L(u)-f(x, t)$. Applying the fractional integral operator ${ }^{\mathrm{CF}} I_{0}^{\alpha}$ yields

$$
u(x, t)-u(x, 0)=\left({ }^{\mathrm{CF}} I_{0}^{\alpha}\right)(L(u)-f)=\frac{1-\alpha}{B(\alpha)}(L(u)-f)+\frac{\alpha}{B(\alpha)} \int_{0}^{t}(L(u)-f) d \tau .
$$

By the continuity of the solution $u$, the above equations hold true at $t=0$, which yields $(L(u)-f)(x, 0)=0$.

Theorem 3.2 Let $u \in C^{2}[0, \ell] \cap H^{1}(0, T]$ be a solution to the time-fractional initialboundary value problem (3.1)-(3.3) with $f(x, 0)=0$. Let $z(t) \in C[0, T]$ be such that

$$
|f(x, t)| \leq z(t), \quad(x, t) \in \bar{\Omega}, z(0)=0,
$$

and $k(t)$ be the unique solution of

$$
\left({ }^{\mathrm{CFC}} D_{0}^{\alpha} k\right)(t)=z(t), \quad k(0)=0 .
$$


Then it holds that

$$
\begin{aligned}
\|u\|_{\bar{\Omega}} \leq & \|k(t)\|_{[0, T]} \\
& +\max \left\{\|g(x)\|_{[0, \ell]}\left\|h_{1}(t)\right\|_{[0, T]}+\|k(t)\|_{[0, T]},\left\|h_{2}(t)\right\|_{[0, T]}+\|k(t)\|_{[0, T]}\right\},
\end{aligned}
$$

where $\|k(t)\|_{[0, T]} \leq \frac{1-\alpha}{B(\alpha)}\left(1+\frac{\alpha}{1-\alpha} T\right)\|z(t)\|_{[0, T]}$.

Proof Since $z(0)=0$, applying the result in Eq. (3.5), we have

$$
\|k(t)\|_{[0, T]} \leq \frac{1-\alpha}{B(\alpha)}\left(1+\frac{\alpha}{1-\alpha} T\right)\|z(t)\|_{[0, T]} .
$$

Let $v(x, t)=u(x, t)-k(t)$, then

$$
\begin{aligned}
P_{\alpha}(v(x, t)) & =L(v(x, t))-\left({ }^{\mathrm{CFC}} D_{0}^{\alpha} v(x, t)\right)(t) \\
& =L(u(x, t))-\left({ }^{\mathrm{CFC}} D_{0}^{\alpha} u(x, t)\right)(t)+\left({ }^{\mathrm{CFC}} D_{0}^{\alpha} k\right)(t) \\
& =f(x, t)+z(t) \geq 0 .
\end{aligned}
$$

The initial and boundary conditions of $v(x, t)$ are

$$
\begin{aligned}
& v(x, 0)=g(x)-k(0)=g(x), \quad x \in S_{2}, \\
& v(0, t)=h_{1}(t)-k(t), \quad v(\ell, t)=h_{2}(t)-k(t), \quad t \in(0, T] .
\end{aligned}
$$

Applying the weak maximum principle, we have

$$
v(x, t) \leq \max _{(x, t) \in S_{1} \cup S_{2} \cup s_{3}}\left\{g(x), h_{1}(t)-k(t), h_{2}(t)-k(t)\right\}
$$

and thus

$$
u(x, t) \leq k(t)+\max _{(x, t) \in S_{1} \cup S_{2} \cup s_{3}}\left\{g(x), h_{1}(t)-k(t), h_{2}(t)-k(t)\right\} .
$$

For $w(x, t)=-u(x, t)$, we have $P_{\alpha}(w(x, t))=-f(x, t)$. Let $v(x, t)=w(x, t)-k(t)$, then $v(x, t)$ satisfies

$$
\begin{aligned}
& P_{\alpha}(v)=P_{\alpha}(w)+\left({ }^{\mathrm{CFC}} D_{0}^{\alpha} k\right)(t)=-f(x, t)+z(t) \geq 0, \quad 0<\alpha<1,(x, t) \in \Omega, \\
& v(x, 0)=-g(x), \quad 0 \leq x \in S_{2}, \\
& v(0, t)=-h_{1}(t)-k(t), \quad v(\ell, t)=-h_{2}(t)-k(t), \quad t \in(0, t] .
\end{aligned}
$$

Applying the simple maximum principle will lead to

$$
-u(x, t)=w(x, t) \leq k(t)+\max _{(x, t) \in S_{1} \cup S_{2} \cup s_{3}}\left\{-g(x),-h_{1}(t)-k(t),-h_{2}(t)-k(t)\right\} .
$$


Combining Eqs. (3.8) and (3.9) will lead to

$$
|u| \leq\|k(t)\|_{[0, T]}+\max \left\{\|g(x)\|_{[0, \ell]},\left\|h_{1}(t)+k(t)\right\|_{[0, T]},\left\|h_{2}(t)+k(t)\right\|_{[0, T]}\right\}
$$

which proves the result.

Corollary 3.1 Let $u \in C^{2}[0, \ell] \cap H^{1}(0, T]$ be a solution to the time-fractional initialboundary value problem (3.1)-(3.3) with $f(x, t)=0$. Then it holds that

$$
\|u\|_{\bar{\Omega}} \leq \max \left\{\|g(x)\|_{[0, \ell]},\left\|h_{1}(t)\right\|_{[0, T]},\left\|h_{2}(t)\right\|_{[0, T]}\right\} .
$$

Proof Since $f(x, t)=0$, we choose $z(t)=0$, and thus the fractional initial value problem

$$
\left({ }^{\mathrm{CFC}} D_{0}^{\alpha} k\right)(t)=0, \quad k(0)=0,
$$

has the unique solution $k(t)=0$, which proves the result.

We have the following stability result.

Theorem 3.3 Let $u_{1}(x, t)$ and $u_{2}(x, t)$ be two solutions of the time-fractional diffusion equation (3.1) that satisfy the same boundary condition (3.2) and the initial conditions $u_{1}(x, 0)=g_{1}(x), u_{2}(x, 0)=g_{2}(x), 0 \leq x \leq \ell$. Then it holds that

$$
\left\|u_{1}(x, t)-u_{2}(x, t)\right\|_{\bar{\Omega}} \leq\left\|g_{1}(x)-g_{2}(x)\right\|_{[0, \ell]} .
$$

Proof Let $v(x, t)=u_{1}-u_{2}$. Then $v(x, t)$ satisfies

$$
\begin{aligned}
& P_{\alpha}(v)=0, \quad 0<\alpha<1,(x, t) \in \Omega, \\
& v(x, 0)=g_{1}(x)-g_{2}(x), \quad x \in S_{2}, \\
& v(0, t)=v(\ell, t)=0, \quad t \in(0, T] .
\end{aligned}
$$

Applying the weak maximum principle, we have $v(x, t) \leq g_{1}(x)-g_{2}(x)$. Applying analogous statements for $-v(x, t)$ will lead to $-v(x, t) \leq g_{2}(x)-g_{1}(x)$. Thus,

$$
|v(x, t)| \leq\left|g_{1}(x)-g_{2}(x)\right|, \quad(x, t) \in \bar{\Omega},
$$

and the result is obtained.

\section{Nonlinear fractional diffusion problems}

We consider the nonlinear time-fractional diffusion equation of the form

$$
N_{\alpha}(u)=\left({ }^{\mathrm{CFC}} D_{0}^{\alpha} u\right)(t)-L(u)-F(x, t, u)=0, \quad 0<\alpha<1,(x, t) \in \Omega,
$$

subject to the initial and boundary conditions (3.2)-(3.3), where $F(x, t, u)$ is a smooth function. We start with the following uniqueness result. 
Theorem 4.1 If $F(x, t, u)$ is nonincreasing with respect to $u$, then the nonlinear timefractional diffusion equation (4.1) subject to the initial and boundary conditions (3.2)-(3.3) possesses at most one solution $u \in C^{2}[0, \ell] \cap H^{1}(0, T]$.

Proof Assume that $u_{1}(x, t)$ and $u_{2}(x, t)$ are two solutions of (4.1) subject to the initial and boundary conditions (3.2)-(3.3), and let $v(x, t)=u_{1}-u_{2}$. Then $v(x, t)$ satisfies

$$
\begin{aligned}
& P_{\alpha}\left((v(x, t))=L(v(x, t))-\left({ }^{\mathrm{CFC}} D_{0}^{\alpha} v(x, t)\right)(t)=F\left(x, t, u_{2}\right)-F\left(x, t, u_{1}\right), \quad(x, t) \in \Omega,\right. \\
& v(x, 0)=0, \quad 0 \leq x \leq \ell, \\
& v(0, t)=v(\ell, t)=0, \quad t \in(0, T] .
\end{aligned}
$$

Applying the mean value theorem to $F(x, t, u)$ yields

$$
F\left(x, t, u_{2}\right)-F\left(x, t, u_{1}\right)=\frac{\partial F}{\partial u}\left(u^{*}\right)\left(u_{2}-u_{1}\right)=-\frac{\partial F}{\partial u}\left(u^{*}\right) v
$$

where $u^{*}=(1-\mu) u_{1}+\mu u_{2}$ for some $0 \leq \mu \leq 1$. Thus,

$$
P_{\alpha}\left((v(x, t))=L(v(x, t))-\left({ }^{\mathrm{CFC}} D_{0}^{\alpha} v(x, t)\right)(t)=-\frac{\partial F}{\partial u}\left(u^{*}\right) v .\right.
$$

Assume by contradiction that $v$ is not identically zero. Then $v$ has either a positive maximum or a negative minimum. At a positive maximum $\left(x_{0}, t_{0}\right) \in \Omega_{T}$, we have

$$
M=v\left(x_{0}, t_{0}\right)>0, \quad v_{x}\left(x_{0}, t_{0}\right)=0, \quad v_{x x}\left(x_{0}, t_{0}\right) \leq 0 .
$$

Because $a\left(x_{0}, t_{0}\right)>0$, we have $\left.L(v(x, t))\right|_{(x, t)=\left(x_{0}, t_{0}\right)} \leq 0$, which together with

$$
\left({ }^{\mathrm{CFC}} D_{0}^{\alpha} v\left(x_{0}, t\right)\right)\left(t_{0}\right)=\frac{B(\alpha)}{1-\alpha} e^{-\frac{\alpha}{1-\alpha} t_{0}}\left(v\left(x_{0}, t_{0}\right)-v\left(x_{0}, 0\right)\right)=\frac{B(\alpha)}{1-\alpha} e^{-\frac{\alpha}{1-\alpha} t_{0}} M>0
$$

will lead to

$$
\left.L(v(x, t))\right|_{(x, t)=\left(x_{0}, t_{0}\right)}-\left({ }^{\mathrm{CFC}} D_{0}^{\alpha} v\left(x_{0}, t\right)\right)\left(t_{0}\right)<0 .
$$

Because $F$ is nonincreasing, we have $\frac{\partial F}{\partial u}\left(u^{*}\right) \leq 0$, and thus

$$
-\frac{\partial F}{\partial u}\left(u^{*}\right) v\left(x_{0}, t_{0}\right)=-\frac{\partial F}{\partial u}\left(u^{*}\right) M \geq 0 .
$$

The results in Eqs. (4.3) and (4.4) contradict Eq. (4.2). Analogous steps will lead to a contradiction if $v$ has a negative minimum. Thus, $v=0$ and $u_{1}=u_{2}$.

Theorem 4.2 Let $u_{1}(x, t)$ and $u_{2}(x, t)$ be two solutions of the time-fractional diffusion equation (4.1) that satisfy the same boundary condition (3.2) and the initial conditions $u_{1}(x, 0)=g_{1}(x), u_{2}(x, 0)=g_{2}(x), 0 \leq x \leq \ell$. If $F(x, t, u)$ is nonincreasing with respect to $u$, then it holds that

$$
\left\|u_{1}(x, t)-u_{2}(x, t)\right\|_{\bar{\Omega}} \leq\left\|g_{1}(x)-g_{2}(x)\right\|_{[0, \ell]} .
$$


Proof Let $v(x, t)=u_{1}-u_{2}$. Then $v(x, t)$ satisfies, see Eq. (4.2),

$$
\begin{aligned}
& P_{\alpha}\left((v(x, t))=L(v(x, t))-\left({ }^{\mathrm{CFC}} D_{0}^{\alpha} v(x, t)\right)(t)=-\frac{\partial F}{\partial u}\left(u^{*}\right) v, \quad(x, t) \in \Omega,\right. \\
& v(x, 0)=g_{1}(x)-g_{2}(x), \quad 0 \leq x \leq \ell \\
& v(0, t)=v(\ell, t)=0, \quad t \in(0, T] .
\end{aligned}
$$

Let

$$
M_{a}=\left\|g_{1}(x)-g_{2}(x)\right\|_{[0, \ell]},
$$

and assume by contradiction that the result of the theorem is not true. That is,

$$
\|v(x, t)\|_{\bar{\Omega}} \not \leq M_{a} .
$$

Then $v$ either has a positive maximum at a point $\left(x_{0}, t_{0}\right) \in \bar{\Omega}$ with $v\left(x_{0}, t_{0}\right)=M_{1}>M_{a}$, or it has a negative minimum at a point $\left(x_{0}, t_{0}\right) \in \bar{\Omega}$ with $v\left(x_{0}, t_{0}\right)=M_{2}<-M_{a}$. If $v\left(x_{0}, t_{0}\right)=$ $M_{1}>M_{a}$, using the initial and boundary conditions of $v$, we have $\left(x_{0}, t_{0}\right) \in \Omega_{T}$. Applying analogous steps in the proof of the previous theorem, we have

$$
\left.L(v(x, t))\right|_{(x, t)=\left(x_{0}, t_{0}\right)}-\left({ }^{\mathrm{CFC}} D_{0}^{\alpha} v\left(x_{0}, t\right)\right)\left(t_{0}\right)<0
$$

and

$$
-\frac{\partial F}{\partial u}\left(u^{*}\right) v\left(x_{0}, t_{0}\right)=-\frac{\partial F}{\partial u}\left(u^{*}\right) M_{a}>0
$$

which contradicts Eq. (4.5). Applying analogous steps will lead to a contradiction for the case of $v\left(x_{0}, t_{0}\right)=M_{2}<-M_{a}$. Thus, $\|v(x, t)\|_{\bar{\Omega}} \leq M_{a}$, which proves the result.

By applying analogous steps in the proof of Lemma 3.2, we have the following necessary condition for the existence of solution of 4.1.

Lemma 4.1 The nonlinear time-fractional equation (4.1) has a solution only if

$$
(L(u))(x, 0)=F(x, 0, u(x, 0)),
$$

where $L(u)=a(x, t) u_{x x}+b(x, t) u_{x}$.

\section{Illustrated examples}

Example 5.1 Consider the time-fractional initial-boundary value problem

$$
\left({ }^{\mathrm{CFC}} D_{0}^{\alpha} u\right)(t)=\left(1-e^{-t}\right) u_{x x}-t e^{-x}, \quad 0<x<1, t>0,
$$

subject to the initial condition $u(x, 0)=g(x), 0<x<1$, and the homogeneous boundary conditions $u(0, t)=u(1, t)=0, t>0$. Now, $|f(x, t)|=t e^{-x} \leq t, 0<x<1$, and thus we choose $z(t)=t$ in Eq. (3.6). We have

$$
\|k(t)\|_{[0, T]} \leq \frac{1-\alpha}{B(\alpha)}\left(1+\frac{\alpha}{1-\alpha} T\right)\|z(t)\|_{[0, T]} \leq \frac{1-\alpha}{B(\alpha)}\left(1+\frac{\alpha}{1-\alpha} T\right) T .
$$


For $T=1$, the last equation yields

$$
\|k(t)\|_{[0, T]} \leq \frac{1}{B(\alpha)} .
$$

Applying the result in (3.7), we have

$$
\|u\|_{\bar{\Omega}} \leq \frac{1}{B(\alpha)}+\max \left\{\|g(x)\|_{[0,1]}, \frac{1}{B(\alpha)}\right\} .
$$

Example 5.2 Consider the nonlinear time-fractional diffusion equation

$$
N_{\alpha}(u)=\left({ }^{\mathrm{CFC}} D_{0}^{\alpha} u\right)(t)-u_{x x}-e^{-u}=0, \quad 0<x<1, t>0 .
$$

Since $F(x, t, u)=-e^{u}$ satisfies $\frac{\partial F}{\partial u}=-e^{-u} \leq 0$, then $F(x, t, u)=-e^{-u}$ is nonincreasing, and the above diffusion equation has at most one solution by virtue of Theorem 4.1. By Lemma 3.2 a necessary condition for the existence of solution of the above diffusion equation is $u_{x x}(x, 0)=e^{-u(x, 0)}$.

\section{Concluding remarks}

We have considered linear and nonlinear fractional diffusion equations with Caputo fractional derivative of non-singular kernel. We have obtained an estimate of the Caputo fractional derivative of non-singular kernel of a function at its extreme points. We then have derived a weak and a strong maximum principles for the linear time-fractional diffusion equation. We have analyzed the solutions of the linear and nonlinear time-fractional diffusion models using the obtained maximum principles. Some examples are presented to illustrate the applicability of the obtained results. The obtained results will lead to better understanding of the time-fractional diffusion models with Caputo fractional derivative of non-singular kernel. In contrast to previous studies on fractional diffusion models with Caputo and Riemann-Liouville fractional derivatives, it is noticed that extra conditions have been imposed to guarantee the existence of solutions to the linear and nonlinear time-diffusion models.

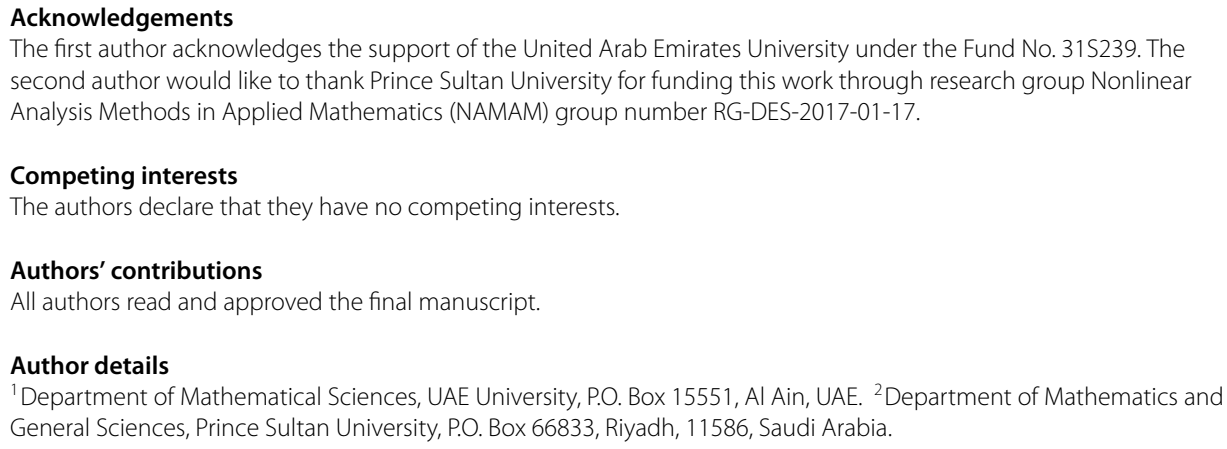

The first author acknowledges the support of the United Arab Emirates University under the Fund No. 31S239. The second author would like to thank Prince Sultan University for funding this work through research group Nonlinear Analysis Methods in Applied Mathematics (NAMAM) group number RG-DES-2017-01-17.

Competing interests

The authors declare that they have no competing interests.

Authors' contributions

All authors read and approved the final manuscript.

Author details

${ }^{1}$ Department of Mathematical Sciences, UAE University, P.O. Box 15551, Al Ain, UAE. ${ }^{2}$ Department of Mathematics and General Sciences, Prince Sultan University, P.O. Box 66833, Riyadh, 11586, Saudi Arabia.

\section{Publisher's Note}

Springer Nature remains neutral with regard to jurisdictional claims in published maps and institutional affiliations. 
References

1. Caputo, M, Fabrizio, M: A new definition of fractional derivative without singular kernel. Prog. Fract. Differ. Appl. 1(2), 73-85 (2015)

2. Atangana, A, Baleanu, D: Caputo-Fabrizio derivative applied to groundwater flow within confined aquifer. J. Eng. Mech. 143(5), D4016005 (2017)

3. Freed, A, Diethelm, K, Luchko, Y: Fractional-order viscoelasticity (FOV): constitutive development using the fractional calculus. NASA's Glenn Research Center, Ohio (2002)

4. Hilfer, R (ed.): Applications of Fractional Calculus in Physics. World Scientific, Singapore (2000)

5. Klages, R, Radons, G, Sokolov, IM (eds.): Anomalous Transport: Foundations and Applications. Wiley-VCH, Weinheim (2008)

6. Luchko, Y, Punzi, A: Modeling anomalous heat transport in geothermal reservoirs via fractional diffusion equations. GEM Int. J. Geomath. 1, 257-276 (2011)

7. Luchko, Y: Fractional Schrödinger equation for a particle moving in a potential well. J. Math. Phys. 54, 012111 (2013)

8. Mainardi, F: Fractional Calculus and Waves in Linear Viscoelasticity. Imperial College Press, London (2010)

9. Sun, HG, Hao, X, Zhang, Y, Baleanu, D: Relaxation and diffusion models with non-singular kernels. Physica A 468, 590-596 (2017)

10. Uchaikin, V: Fractional Derivatives for Physicists and Engineers. Volume I Background and Theory, Volume II Applications. Springer, Heidelberg (2012)

11. Protter, MH, Weinberger, HF: Maximum Principles in Differential Equations. Springer, Berlin (1999)

12. Pucci, $P$, Serrin, JB: The Maximum Principle. Birkhäuser, Basel (2007)

13. Al-Refai, M, Hajji, M: Monotone iterative sequences for nonlinear boundary value problems of fractional order. Nonlinear Anal. 74, 3531-3539 (2011)

14. Al-Refai, M: Basic results on nonlinear eigenvalue problems of fractional order. Electron. J. Differ. Equ. 2012, 1 (2012)

15. Al-Refai, M, Luchko, Y: Maximum principles for the fractional diffusion equations with the Riemann-Liouville fractional derivative and their applications. Fract. Calc. Appl. Anal. 17, 483-498 (2014)

16. Al-Refai, M, Luchko, Y: Maximum principle for the multi-term time-fractional diffusion equations with the Riemann-Liouville fractional derivatives. Appl. Math. Comput. 257, 40-51 (2015)

17. Abdulla, AB, Al-Refai, M, Al-Rawashdeh, A: On the existence and uniqueness of solutions for a class of non-linear fractional boundary value problems. J. King Saud Univ., Sci. 28, 103-110 (2016)

18. Al-Refai, M, Luchko, Y: Analysis of fractional diffusion equations of distributed order: maximum principles and its applications, Analysis 36(2), 123-133 (2015). doi:10.1515/anly-2015-5011

19. Luchko, Y: Maximum principle for the generalized time-fractional diffusion equation. J. Math. Anal. Appl. 351, 218-223 (2009)

20. Luchko, Y: Boundary value problems for the generalized time-fractional diffusion equation of distributed order. Fract. Calc. Appl. Anal. 12, 409-422 (2009)

21. Luchko, Y: Some uniqueness and existence results for the initial-boundary-value problems for the generalized time-fractional diffusion equation. Comput. Math. Appl. 59, 1766-1772 (2010)

22. Luchko, Y: Initial-boundary-value problems for the generalized multi-term time-fractional diffusion equation. J. Math. Anal. Appl. 374, 538-548 (2011)

23. Zhou, Y: Basic Theory of Fractional Differential Equations. World Scientific, Singapore (2014)

24. Ye, H, Liu, F, Anh, V, Turner, I: Maximum principle and numerical method for the multi-term time-space Riesz-Caputo fractional differential equations. Appl. Math. Comput. 227, 531-540 (2014)

25. Eidelman, SD, Kochubei, AN: Cauchy problem for fractional diffusion equations. J. Differ. Equ. 199, $211-255$ (2004)

26. Kochubei, AN: Fractional-order diffusion. Differ. Equ. 26, 485-492 (1990)

27. Abdeljawad, T, Baleanu, D: On fractional derivatives with exponential kernel and their discrete versions. Rep. Math. Phys. 80(1), 11-27 (2017)

28. Abdeljawad, T, Baleanu, D: Monotonicity analysis of a nabla discrete fractional operator with discrete Mittag-Leffler kernel. Chaos Solitons Fractals 102, 106-110 (2017)

29. Abdeljawad, T, Baleanu, D: Monotonicity results for fractional difference operators with discrete exponential kernels. Adv. Differ. Equ. 2017, 78 (2017). doi:10.1186/s13662-017-1126-1

30. Atangana, A, Baleanu, D: New fractional derivative with non-local and non-singular kernel. Therm. Sci. 20(2), 757-763 (2016)

31. Abdeljawad, T, Baleanu, D: Discrete fractional differences with nonsingular discrete Mittag-Leffler kernels. Adv. Differ. Equ. 2016, 232 (2016). doi:10.1186/s13662-016-0949-5

32. Abdeljawad, T, Baleanu, D: Integration by parts and its applications of a new nonlocal fractional derivative with Mittag-Leffler nonsingular kernel. J. Nonlinear Sci. Appl. 10(3), 1098-1107 (2017)

33. Al-Refai, M: On the fractional derivative at extreme points. Electron. J. Qual. Theory Differ. Equ. 2012,55 (2012) 